\title{
Novel Joint Subcarrier and Power Allocation Method in SWIPT for WSNs Employing OFDM System
}

\author{
Saleemullah Memon ${ }^{1}$ \\ School of Information \& Communication Engineering \\ Beijing University of Posts and Telecommunications \\ Beijing, China
}

\author{
Kamran Ali Memon*2 \\ State Key Laboratory of Information Photonics and Optical \\ Communications (IPOC), School of Electronic Engineering \\ Beijing University of Posts and Telecommunications \\ Beijing, China \\ Zulfiqar Ali Zardari ${ }^{3}$ \\ Faculty of Information Technology \\ Beijing University of Technology, Beijing, China
}

\author{
Muhammad Aamir Panhwar ${ }^{4}$ \\ School of Electronic Engineering \\ Beijing University of Posts and Telecommunications \\ Beijing, China
}

\begin{abstract}
Sijjad Ali Khuhro ${ }^{5}$
School of Computer Science and technology

University of Science and technology of china

China
\end{abstract}

\begin{abstract}
In recent research trends, simultaneous wireless information and power transfer (SWIPT) has proved to be an innovative technique to deal with limited energy problems in energy harvesting (EH) technologies for wireless sensor networks (WSNs). In this paper, a method of subcarrier and power allocation for both EH and information decoding (ID) operations is proposed under orthogonal frequency division multiplexing (OFDM) systems, with an improved the quality of service (QoS) parameters. This proposed method assigns one group of subcarriers for ID and remaining group of subcarriers is assigned for EH, despite of applying any splitting schemes. We achieved maximum EH under the ID and power constraints with an effective algorithm for the first time incorporating the dual decomposition technique which deals with power and subcarrier allocation problem jointly. The obtained simulation outcomes in relation to power allocation ratio, subcarrier allocation ratio and energy harvested $(\mathrm{EH})$ at the destination node proved better when compared to the schemes that contain water filling, time switching (TS) or power splitting (PS) approaches under different target transmission rates and transmitter and receiver distances.
\end{abstract}

Keywords-Simultaneous wireless information \& power transfer (SWIPT); Energy harvesting (EH); Information decoding (ID); power allocation; subcarrier allocation

\section{INTRODUCTION}

The concept of simultaneous transformation of information and energy in the field of wireless communication has recently received an upsurge of interest for the researchers. Through this simultaneous wireless information and power transfer (SWIPT), mobile phone users have access to more than just energy but data as well at the same time, which provides huge prospects. However, some ultimate strategic variations are needed in wireless communication networks to bring SWIPT for efficient applications [1]. The rates of transmitting

\footnotetext{
*Corresponding Author
}

information and the reliability of reception are suitably applied to estimate the wireless systems performance [2].

In [2], an impractical receiver is considered, which possesses the capability to achieve energy harvesting (EH) and information decoding (ID) concurrently. Furthermore, the agreement between harvesting energy and information rate level are essential to determine the system pursuance when the receiver carryout harvested energy using radio frequency (RF) signal [2]. In [3] and [4], however, two constructive SWIPT power splitting (PS) and time switching (TS) strategies were considered. The PS scheme divides the signal received by receiver into two parts with distinct powers, one part is assigned for ID operation and the other part is assigned for EH operation. Whereas in the TS strategy, the receiver automatically switches within one transmission time to EH or ID mode. In addition to the receiver circuit, the PS design of conventional communication systems [5] does not require any further changes. In [6], the major problem of efficient energy optimization in the multi-input-single-output (MISO) downlink scheme based on the PS scheme was considered. This method used langrangian relaxation in conjunction with the dinkelbach method, which maximized the energy efficiency under the constraints of $\mathrm{EH}$ and signal-to-interference-plus-noise-ratio (SINR).

Preliminary research is based on point-to-point multi-inputmulti-output (MIMO) connections that actually communicate with each other via two devices with numerous antennas. However, current focus has indeed moved to multiuser MIMO systems, where a group of single-antenna users are concurrently assisted by the base station equipped with multiantennas [7]. In the classic work of [8], SWIPT was investigated by using TS and PS schemes in massive MIMO 
which can allow multi-way relay networks (MWRNs) and energy restricting amplify and forward relays.

Orthogonal frequency division multiplexing (OFDM) is a commonly used multi-carrier method in different wireless standards. The overall performance of OFDM based SWIPT mechanisms has been studied in many existing papers [9]-[10]. In order to analyze the performance of SWIPT, [9] selected an OFDM based multi user single-antenna system with a PS technique at the receiver. The results conclude that the energy efficiency of the system can be greatly improved by using RF $\mathrm{EH}$ in the limited interference regime.

Multi-antenna receivers are useful for improving the system potential capacity rather than improving the energy efficiency of the system [9]. Authors in [11] focused on SWIPT for broadband wireless systems (BWSs) involving OFDM and beamforming transition. This can develop a number of parallel sub channels to simply the mechanism of the resource allocation. Carriers in [11] are used for EH proposed for that specific user by employing a fixed subcarrier allocation. However, when it comes to multi-user wireless systems with OFDM, authors in [12] researched on the phenomenon of multi distributed users receiving a broadcasted message from a single fixed access point. The authors in [12] considers two multiple access schemes in their analysis, notably time division multiple access (TDMA) and multiple orthogonal frequency division access (OFDMA). TDMA employs TS approach where information receiver for a user works for scheduled period whereas its energy receiver operates for all the periods. However, OFDMA assumes that available subcarriers share the same PS ratio at the receiver. Author in [10] presents the analysis of SWIPT receivers with a single OFDM user channel with an upper limit for the rate of energy tradeoff system under consideration. Simulation results conclude the trade-off between the achievable rate and the harvested energy which serves to be a well-known trade-off between the rate and energy of the system.

In WSNs, small nodes are jot usual near to each other which may adversely affect each other due to urban, climatic or geographical obstacles. As a result, Line of sight (LOS) communication becomes impracticable, therefore [13] and [14] suggests to use idle nodes for creating intermediate hops. Relaying consumes some extra energy to serve the purpose of cooperative transmission. Relaying protocols work in two fashions i.e. a) Decode then forward or regenerate and b) Amplify then forward or transparent relaying as reported in [15] and [16]. [17] analyzes a three-node scheme and comes up with an efficient resource allocation in terms of throughput and energy usage. The same is repeated for multiple access relay arrangement, as found in [18] and [19]. [20] extends it with multi antenna use and utilizes harvested energy not for storing in batteries but for the operational needs. Furthermore, [20] presents a tradeoff for the EH time and the time period relay node is communicating. Author in [21] introduces the methods to reduce interference in RF-EH operation for such mentioned relaying systems.
Lot of research had been focused on SWIPT in an OFDM comprised of PS or TS schemes, where destination node requires a time or power divider to distinguish the signal for ID operation and EH operation. In our work, we have considered SWIPT in joint power and subcarrier allocation-based architecture in an OFDM system, where have not considered any separate splitter/divider at the receiving end. Particularly, the OFDM based subcarriers are partitioned in two portions. One portion is allocated for one group that is used for $\mathrm{EH}$ and the other potion is allocated for another group that is used for ID of the received signal. Hence, the only job of receiving node is to consider which group is assigned for the operation of $\mathrm{EH}$, at that time other group will be assigned for the operation of ID. Thus, at the receiving node no splitter/divider is needed anymore. The key contributions of this work can be labelled as:

- $\mathrm{EH}$ is maximized under the allocation of joint power and subcarrier criteria, ensuring the achievable target transmission rates.

- The complexity of receiver is minimized in proposed work and no splitter is used as compared to the previous work of SWIPT in OFDM schemes.

- Simulation results validate the dominance of our proposed joint power and subcarrier allocation scheme when compared to the schemes that contain water filling or PS/TS approaches.

The remaining organization of this paper is given as follows. Section 2 presents the system model of our proposed work. Section 3 explains the problem formulation of $\mathrm{EH}$ optimization and joint optimal power and subcarrier allocation problems. Section 4 provides the solution of the optimization problems. Section 5 presents the performance of our proposed work. Finally, Section 6 concludes the overall research work and contains future work as well.

\section{SYSTEM MODEL}

A wireless link based on OFDM, consists of a single antenna transmitter (Tx) and a single antenna receiver ( $\mathrm{Rx})$, as revealed in Fig. 1. We represented all the subcarriers as $\mathrm{I}=\{1,2, \ldots, \mathrm{J}\}$ and the total bandwidth of the system is divided equally into J subcarriers.

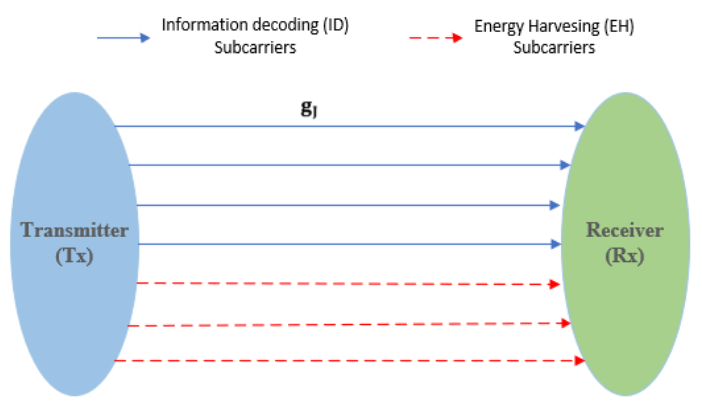

Fig. 1. Wireless SWIPT Architecture. 
We have assumed that channel power gain is perfectly known at the transmitter and is indicated as $\mathrm{g}_{\mathrm{J}}$. Let $\mathrm{P}_{\mathrm{t}}$ denotes the total transmitted power to all $\mathrm{J}$ subcarriers and $\mathrm{p}_{\mathrm{J}}$ indicates the power allocated to subcarrier J. We have assumed a slow fading situation where all the constant channels coefficients are considered. A bandpass filter is assumed at the receiving node. On each $\mathrm{J}$ subcarrier, the received signal corrupted by the noise $\mathrm{n}_{\mathrm{J}}$ is designed as an additive-white-Gaussian-noise (AWGN) having 0 mean with a variance of $\sigma_{\mathrm{J}}^{2}$ and is given as $\mathrm{n}_{\mathrm{J}} \sim \mathcal{C} \mathcal{N}\left(0, \sigma_{\mathrm{J}}^{2}\right)$.

\section{ENERgy HARVESTING OPTIMIZATION PROBLEM}

Based on the SWIPT framework, the signal received at Rx is partitioned into two groups, one group is for the process of ID and the other is for the process of EH operation over all the subcarriers, as given below.

$\mathrm{I}=\mathrm{K}_{\mathrm{ID}}+\mathrm{K}_{\mathrm{EH}}$

where $\mathrm{K}_{\mathrm{ID}} \leq \mathrm{I}$ and $\mathrm{K}_{\mathrm{EH}} \leq \mathrm{I}$ denote the subcarriers used for ID and EH respectively. The EH at the Rx node is given as

$\mathrm{E}=\xi \sum_{\mathrm{J} \in \mathrm{K}_{\mathrm{EH}}}\left(\mathrm{p}_{\mathrm{J}} \mathrm{g}_{\mathrm{J}}+\sigma_{\mathrm{J}}^{2}\right)$

where $\xi$ represents the conversion efficiency of energy at $\mathrm{Rx}$, it is assumed that $\xi=1$, for convenience. For the OFDM link, transmission rate can be achieved as

$\mathrm{R}_{\mathrm{T}}=\sum_{\mathrm{J} \in \mathrm{K}_{\mathrm{ID}}} \log _{2}\left(1+\mathrm{p}_{\mathrm{J}} \mathrm{g}_{\mathrm{J}} / \sigma_{\mathrm{J}}^{2}\right)$

In order to satisfy the constraints of power and target date rate requirement, we aim to maximize the $\mathrm{EH}$ under joint subcarrier and power allocation. Thus, the optimization problem can be formulated as

$$
\begin{array}{ll}
\max _{\text {s.t }} \quad & \mathrm{E} \\
\sum_{\mathrm{J} \in \mathrm{K}_{\mathrm{EH}}} \mathrm{p}_{\mathrm{J}}+\sum_{\mathrm{J} \in \mathrm{K}_{\mathrm{ID}}} \mathrm{p}_{\mathrm{J}} \leq \mathrm{P}_{\mathrm{t}}
\end{array}
$$

where $\Psi$ denotes the minimum target transmission rate requirement.

\section{OPTIMIZATION SOLUTION}

The EH optimization problem mentioned above is a nonconvex mixed integer problem; hence it is infeasible to find the direct solution of such problems because of high complexity. However, considering large number of subcarriers, the timing sharing condition can be applied [22]-[23], which makes the duality gap to zero. Thus, the optimization problem can be solved using dual decomposition method. The Lagrange dual function is given as

$\Gamma(\lambda)=\max _{\{\mathbb{P}, \mathbb{K}\}} \mathcal{L}(\mathbb{P}, \mathbb{K})$

where

$$
\begin{gathered}
\mathcal{L}(\mathbb{P}, \mathbb{K})=\mathrm{E}+\lambda_{1}\left(\mathrm{R}_{\mathrm{T}}-\Psi\right)+ \\
\lambda_{2}\left(\mathrm{P}_{\mathrm{t}}-\sum_{\mathrm{J} \in \mathrm{K}_{\mathrm{EH}}} \mathrm{p}_{\mathrm{J}}-\sum_{\mathrm{J} \in \mathrm{K}_{\mathrm{ID}}} \mathrm{p}_{\mathrm{J}}\right)
\end{gathered}
$$

$\mathbb{P}=\left\{\mathrm{p}_{\mathrm{J}}\right\}$ and $\mathbb{K}=\left\{\mathrm{K}_{\mathrm{ID}}, \mathrm{K}_{\mathrm{EH}}\right\}$ denote the power and subcarrier allocation set respectively and $\lambda=\left(\lambda_{1}, \lambda_{2}\right)$ denotes the dual variable vector having positive value subject to rate and power constraints. The dual optimization problem can be obtained as

$\min _{\lambda} \Gamma(\lambda)$

s.t $\quad \lambda \geq 0$

As the dual function is a convex as proved in [11], so in order to minimize the lagrange dual function $\Gamma(\lambda)$, we employ sub-gradient based method that will assure the convergence. The sub-gradient method is given as

$\Delta \lambda_{1}=\mathrm{R}_{\mathrm{T}}-\Psi$

$\Delta \lambda_{2}=\mathrm{P}_{\mathrm{t}}-\sum_{\mathrm{J} \in \mathrm{K}_{\mathrm{EH}}} \mathrm{p}_{\mathrm{J}}-\sum_{\mathrm{J} \in \mathrm{K}_{\mathrm{ID}}} \mathrm{p}_{\mathrm{J}}$

$\Gamma(\lambda)$ cannot be obtained without optimal $\mathbb{P}$ and $\mathbb{K}$ at given values of $\lambda$. We define a two-step process in order to obtain optimal $\mathbb{P}$ and $\mathbb{K}$. In the first step we achieve the optimal $\mathbb{P}$ while fixing, in the later step we find the optimal $\mathbb{K}$.

\section{A. Optimal $\mathbb{P}$ while fixing $\mathbb{K}$}

For a fixed $\mathbb{K}$, partially derivate (6) with respect to variables of optimization problem $\mathrm{p}_{\mathrm{J}}$, can be expressed as follow

$\frac{\partial \mathcal{L}(\mathbb{K}, \mathbb{P})}{\partial \mathrm{p}_{\mathrm{J}}}=\xi \mathrm{g}_{\mathrm{J}}-\lambda_{2}, \quad J \in \mathrm{K}_{\mathrm{EH}}$
$\frac{\partial \mathcal{L}(\mathbb{K}, \mathbb{P})}{\partial \mathrm{p}_{\mathrm{J}}}=\frac{\lambda_{1} \mathrm{~g}_{\mathrm{J}}}{\mathrm{p}_{\mathrm{J}} \mathrm{g}_{\mathrm{J}}+\sigma_{\mathrm{J}}^{2}}-\lambda_{2}, \quad J \in \mathrm{K}_{\mathrm{ID}}$

After applying the Kuhn-Karush-Tucker (KKT) conditions, the partial derivatives of the Lagrange tend to zero, hence the optimal $\mathrm{p}_{\mathrm{J}}\left(J \in \mathrm{K}_{\mathrm{EH}}\right)$ for a given $\lambda$, can be expressed as

$\mathrm{p}_{\mathrm{J}}^{*}=\mathrm{p}_{\max }=\xi \mathrm{g}_{\mathrm{J}}>\lambda_{2}$

$\mathrm{p}_{\mathrm{J}}^{*}=\mathrm{p}_{\min }=\xi \mathrm{g}_{\mathrm{J}} \leq \lambda_{2}$

The optimal $\mathrm{p}_{\mathrm{J}}\left(\mathrm{J} \in \mathrm{K}_{\mathrm{ID}}\right)$ for a given $\lambda$, can be expressed as

$\mathrm{p}_{\mathrm{J}}^{*}=\left(\frac{\lambda_{1}}{\lambda_{2}}-\frac{\sigma_{\mathrm{I}}^{2}}{\mathrm{~g}_{\mathrm{J}}}\right)^{+}$

where, $P_{\max }$ and $P_{\min }$ are maximum and minimum constraints of power respectively on each subcarrier.

\section{B. Optimal $\mathbb{K}$}

After achieving optimal $\mathrm{P}$, optimal $\mathrm{K}$ can be obtained by substituting the optimal (12), (13) and (14) in (6), after rearranging, the Lagrange can be rewritten as

$$
\begin{aligned}
& \mathcal{L}(\mathbb{K})=\sum_{\mathrm{J} \in \mathrm{K}_{\mathrm{ID}}}\left(\lambda_{1} \log _{2}\left(1+\mathrm{p}_{\mathrm{J}}^{*} \mathrm{~g}_{\mathrm{J}} / \sigma_{\mathrm{J}}^{2}\right)-\xi\left(\mathrm{p}_{\mathrm{J}}^{*} \mathrm{~g}_{\mathrm{J}}+\sigma_{\mathrm{J}}^{2}\right)\right)+ \\
& \sum_{\mathrm{J}=1}^{\mathrm{J}}\left(\xi\left(\mathrm{p}_{\mathrm{J}}^{*} \mathrm{~g}_{\mathrm{J}}+\sigma_{\mathrm{J}}^{2}\right)-\lambda_{2} \mathrm{p}_{\mathrm{J}}^{*}\right)-\lambda_{1} \Psi+ \\
& \lambda_{2} \mathrm{P}_{\mathrm{t}}
\end{aligned}
$$

From (15), we can be seen that only right-side portion of the equation contains the subcarriers for ID, hence separating (15) as,

$$
\mathrm{F}_{\mathrm{J}}^{*}=\lambda_{1} \log _{2}\left(1+\mathrm{p}_{\mathrm{J}}^{*} \mathrm{~g}_{\mathrm{J}} / \sigma_{\mathrm{J}}^{2}\right)-\xi\left(\mathrm{p}_{\mathrm{J}}^{*} \mathrm{~g}_{\mathrm{J}}+\sigma_{\mathrm{J}}^{2}\right)
$$


The resultant set of optimum subcarriers $\mathrm{K}_{\mathrm{ID}}$ that will maximize the Lagrange dual function. Consequently, the optimum $\mathrm{K}_{\mathrm{ID}}$ can be obtained as

$\mathrm{K}_{\mathrm{ID}}^{*}=\arg \max _{\mathrm{K}_{\mathrm{ID}}} \sum_{\mathrm{J} \in \mathrm{K}_{\mathrm{ID}}} \mathrm{F}_{\mathrm{J}}^{*}$

From the set subcarriers, the remaining optimal $\mathrm{K}_{\mathrm{EH}}$ can be obtained as

$\mathrm{K}_{\mathrm{EH}}^{*}=\mathrm{I}-\mathrm{K}_{\mathrm{ID}}^{*}$

Hence, the primal optimization variables $\mathbb{P}$ and $\mathbb{K}$ are successfully achieved with the help of specified dual variables. Therefore, the mentioned optimum problem in (4) can now be completely solved by the process of updating the values of primal dual variables.

\section{SimUlation RESUlts AND DisCUSSIONS}

This section presents the simulation results and improvements achieved for joint resource allocation in an OFDM based SWIPT architecture as compared to previous research works. We consider power allocation ratio, subcarrier allocation ratio and energy harvested $(\mathrm{EH})$ at the destination node as the evaluation matrices. For the fading channel of frequency selection, we have used 6 taps and kept central frequency at $1.9 \mathrm{GHz}$. This research in this paper is limited to small scale fading scenarios. The primary role is the LOS signal then the Rician fading channel is designed. Particularly, for all the subcarriers the channel modeling is given as

$f(J)=\sqrt{\frac{N}{1+N}} \tilde{f}+\sqrt{\frac{1}{1+N}} \hat{f}(J)$

The channel power-gain is represented as $g_{J}=|f(J)|^{2}$. The limitations and complete list of parameters used during our simulations are presented in the Table I.

Fig. 2 shows the harvested energy (EH) vs. power transmitted $\left(\mathrm{P}_{\mathrm{t}}\right)$ at different target transmission rates $(\Psi)$. We can observe that as the requirements of $\Psi$ increases in result less energy is harvested. The reason is that with high target rate more power is needed for decoding the ID process wherever the transmitted power is fixed. Consequently, less power will remain for $\mathrm{EH}$ operation.

TABLE I. VARIABLES AND VALUES OF THE PARAMETERS USED

\begin{tabular}{|l|l|}
\hline LOS deterministic component & $\tilde{\boldsymbol{f}}$ \\
\hline Rayleigh fading component & \multicolumn{1}{|c|}{$\hat{\mathrm{f}}(\mathrm{J})$} \\
\hline Rician fading channel & $\mathrm{f}(\mathrm{J})$ \\
\hline No. of subcarriers $(\mathrm{I})$ & 32 \\
\hline Rician factor $(\mathrm{N})$ & 3 \\
\hline Noise spectrum density & $-45 \mathrm{dBm}$ \\
\hline Energy Conversion Efficiency $(\xi)$ & $100 \%(\boldsymbol{\xi}=\mathbf{1})$ \\
\hline Tx \& Rx distance, d & $4 \mathrm{~m}$ \\
\hline Target transmission rate $(\Psi)$ & $5 \mathrm{bps} / \mathrm{Hz}$ \\
\hline
\end{tabular}

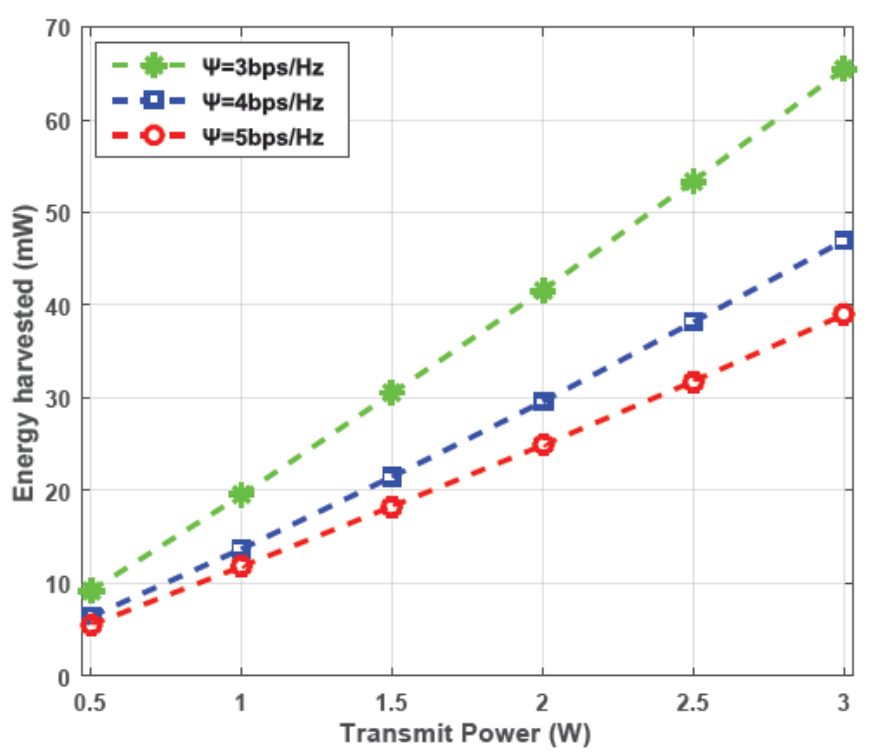

Fig. 2. $\quad E H$ vs. $P_{t}$ at different $\Psi$.

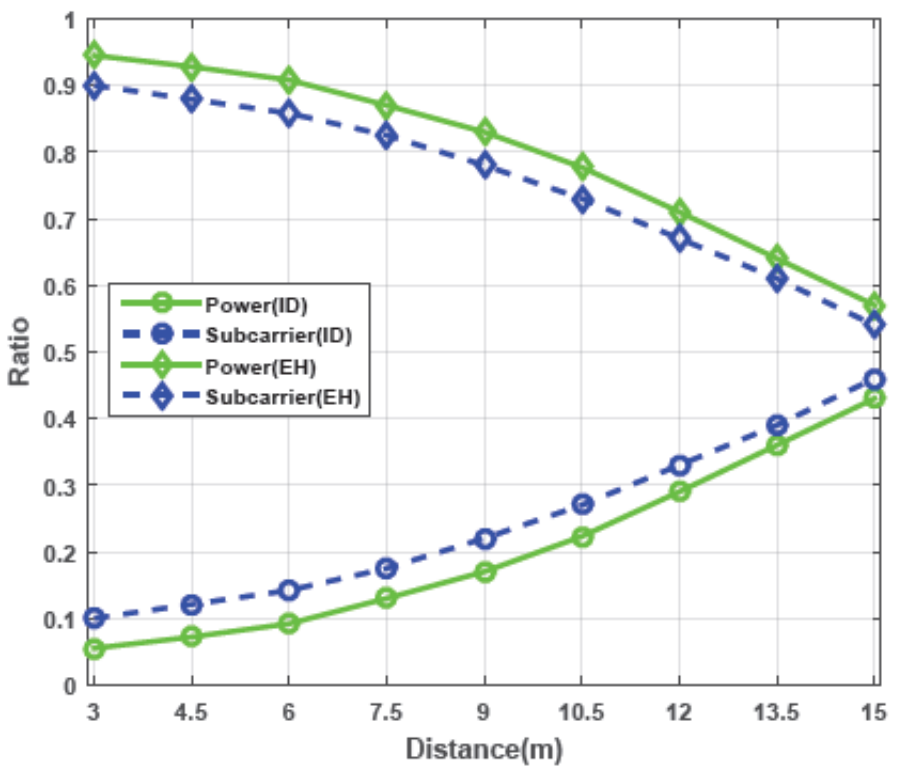

Fig. 3. Subcarrier/Power Allocation Ratio vs. Tx and Rx Distance.

Fig. 3 shows the variations in allocation of power ratios and allocation of subcarrier ratios in relation to separation distance between Tx and Rx, where the value of transmitted power is fixed to $0.5 \mathrm{~W}$. We can observe from Fig. 3 that as the distance between the $\mathrm{Tx}$ and $\mathrm{Rx}$ increase, the more subcarriers and powers are allocated for decoding the information process and less subcarriers and powers are allocated for harvesting the energy process at the same time. This is because, when the distance increases, the channel between the Tx and Rx deteriorates, consequently more resources are assigned for ID operation to meet with the fixed target rate. Thus, few subcarriers and less powers are left for $\mathrm{EH}$ operation. 


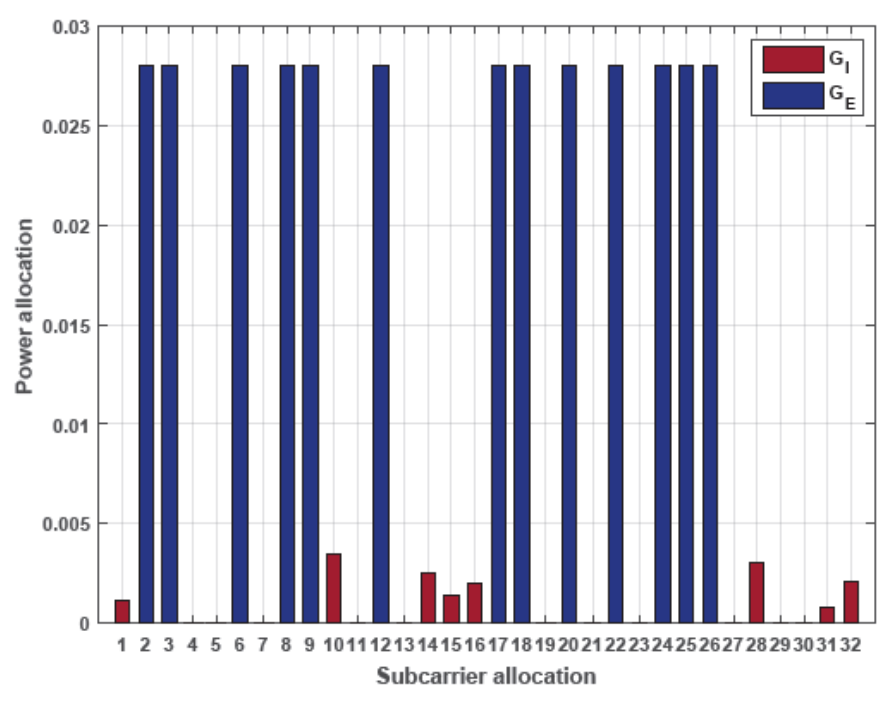

Fig. 4. Subcarrier and Power Allocation at $\Psi=5 \mathrm{bps} / \mathrm{Hz}$.

Fig. 4 display the joint resource (power/subcarrier) allocations at target transmission rate $\Psi=5 \mathrm{bps} / \mathrm{Hz}$ when $\mathrm{P}_{\mathrm{t}}=0.5 \mathrm{~W}$ and $\mathrm{d}=0.6 \mathrm{~m}$. We can conclude that most portion of the resources are assigned for the operation of $\mathrm{EH}$. The reason is that low target rate values require very low portions of resource allocation in order to take part for the ID process.

\section{CONCLUSION AND FUTURE WORK}

We have proposed a joint power and subcarrier allocation method based on SWIPT framework under OFDM architecture, where a separate splitter is not considered at the receiving node. Particularly, the OFDM subcarriers are partitioned in two portions. One portion is allocated for one group that is used for $\mathrm{EH}$ and the other potion is allocated for another group that is used for ID of the received signal. The receiving node only needs to consider which group is allocated for $\mathrm{EH}$, then the other group will be allocated for ID. EH is maximized under the allocation of joint power and subcarrier criteria, though ensuring the achievable target transmission rate. Simulation results show that advantages of our proposed SWIPT OFDM strategy and reveal the dominance of our proposed joint power and subcarrier allocation scheme compared to the schemes that contain water filling or PS/TS approaches.

Furthermore, for future work, researchers can investigate some appropriate techniques to permit the operation of vacant subcarriers for EH and ID. In this situation, multiple frequency bands would be needed so that SWIPT receiver can be able to perform the suitable operations.

\section{REFERENCES}

[1] T. Perera, D. N. Jayakody, S. K. Sharma, S. Chatzinotas, and J. Li, Simultaneous Wireless Information and Power Transfer (SWIPT): Recent Advances and Future Challenges, vol. PP. 2018.

[2] L. R. Varshney, Transporting Information and Energy Simultaneously. 2008.

[3] L. Liu, R. Zhang, and K. Chua, Wireless Information Transfer with Opportunistic Energy Harvesting, vol. 12. 2012.

[4] L. Liu, R. Zhang, and K. Chua, Wireless Information and Power Transfer: A Dynamic Power Splitting Approach, vol. 61. 2013.
[5] X. Zhou, Training-Based SWIPT: Optimal Power Splitting at the Receiver, vol. 64. 2014.

[6] Q. Shi, C. Peng, W. Xu, M. Hong, and Y. Cai, Energy efficiency optimization for MISO SWIPT systems with zero-forcing beamforming, vol. 64. 2015.

[7] lu lu, G. Li, A. Swindlehurst, A. Ashikhmin, and R. Zhang, An Overview of Massive MIMO: Benefits and Challenges, vol. 8. 2014.

[8] R. Zhang and C. K. Ho, MIMO Broadcasting for Simultaneous Wireless Information and Power Transfer., vol. 12. 2011.

[9] D. W. K. Ng, E. S. Lo, and R. Schober, Wireless Information and Power Transfer: Energy Efficiency Optimization in OFDMA Systems, vol. 12. 2013.

[10] P. Grover and A. Sahai, Shannon meets Tesla: Wireless information and power transfer. 2010.

[11] K. Huang and E. G. Larsson, Simultaneous Information and Power Transfer for Broadband Wireless Systems, vol. 61. 2012.

[12] X. Zhou, R. Zhang, and C. K. Ho, Wireless Information and Power Transfer in Multiuser OFDM Systems, vol. 13. 2013.

[13] M. Peng, Y. Liu, D. Wei, W. Wang, and H.-H. Chen, Hierarchical cooperative relay based heterogeneous networks, vol. 18. 2011.

[14] B. Zhou, H. Hu, S.-Q. Huang, and H.-H. Chen, Intracluster Device-toDevice Relay Algorithm With Optimal Resource Utilization, vol. 62. 2013.

[15] J. Nicholas Laneman and G. W. Wornell, Energy-Efficient Antenna Sharing and Relaying for Wireless Networks, vol. 1. 2000.

[16] J. Nicholas Laneman, D. N. C. Tse, and G. W. Wornell, Cooperative Diversity in Wireless Networks: Efficient Protocols and Outage Behavior, vol. 50. 2005.

[17] H. (Henry Chen, Y. Li, J. Luiz Rebelatto, B. F. Uchoa-Filhoand, and B. Vucetic, Harvest-Then-Cooperate: Wireless-Powered Cooperative Communications, vol. 63. 2014.

[18] A. Rajaram, D. N. Jayakody, and V. Skachek, Store-then-cooperate: Energy harvesting scheme in cooperative relay networks. 2016.

[19] W. Huang, H. (Henry Chen, Y. Li, and B. Vucetic, On the Performance of Multi-Antenna Wireless-Powered Communications with Energy Beamforming, vol. 65. 2015.

[20] I. Krikidis, G. Zheng, and B. Ottersten, Harvest-use cooperative networks with half/full-duplex relaying. 2013.

[21] A. Nasir, X. Zhou, S. Durrani, and R. Kennedy, Relaying Protocols for Wireless Energy Harvesting and Information Processing, vol. 12. 2012.

[22] Y. Liu and X. Wang, Information and Energy Cooperation in OFDM Relaying: Protocols and Optimization, vol. 65. 2015.

[23] W. Yu and R. Lui, Dual Methods for Nonconvex Spectrum Optimization of Multicarrier Systems., vol. 54. 2006.

\section{AUTHOR's PROFILE}

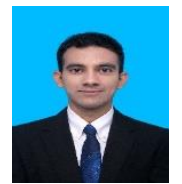

Saleemullah Memon received his B.E degree in electronic engineering from Quaid-e-Awam University of Engineering, Science and Technology (QUEST), Pakistan, in 2017. He is currently pursuing his MS degree at Key Laboratory of Universal Wireless Communication (Ministry of Education), Beijing University of Posts and Telecommunications (BUPT), China. His current research interests include wireless communication and networks, simultaneous wireless information and power transfer (SWIPT) in cooperative relaying networks and MIMO systems.

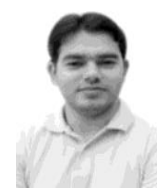

Kamran Ali Memon received his Bachelor Degree in Electronics Engineering (2009) from Mehran University of Engineering Technology, Jamshoro Pakistan and Master's Degree in Communication (2015) from Quaid e Awam UEST Nawabshah Pakistan. He worked as a Lecturer/Assistant Professor in QUEST Pakistan for 08 years. Currently he is working toward his $\mathrm{PhD}$ at State Key Laboratory of Information Photonics and Optical Communications (IPOC), Beijing University of Posts and telecommunications, China. His research interests include optical and Wireless communications, PONs, Radio over fiber and WSNs. 